\title{
The soliton solutions for semidiscrete complex mKdV equation
}

\author{
Corina N. Babalic ${ }^{1, *}$ \\ ${ }^{1}$ University of Craiova, 13 A.I. Cuza, 200585, Craiova, Romania
}

\begin{abstract}
The semidiscrete complex modified Korteweg-de Vries equation (semidiscrete $\mathrm{cmKdV}$ ), which is the second member of the semidiscrete nonlinear Schrődinger hierarchy (Ablowitz-Ladik hierarchy), is solved using the Hirota bilinear formalism. Starting from the focusing case of semidiscrete form of $\mathrm{cmKdV}$, proposed by Ablowitz and Ladik, we construct the bilinear form and build the multi-soliton solutions. The complete integrability of semidiscrete $\mathrm{cmKdV}$, focusing case, is proven and results are discussed.
\end{abstract}

\section{Introduction}

The complex modified Korteweg-de Vries (cmKdV) equation has attracted a lot of attention due to the wide range of physical applications in fields like nematic optical fibers [1], few-cycle optical pulses [2] and also due to the important results obtained such as conserved quantities, stability of solitary wave solutions, numerical simulations, symmetry constraints, Darboux transformation, and various solutions [3-6]. The first discrete version of $\mathrm{cmKdV}$ has been proposed in 1976 by Ablowitz and Ladik [7] and during the last decades interesting investigations have been made also on other versions of differential-difference complex mKdV in [8-12]: Lax pair, conservation laws, numerical simulations, N-fold Darboux transformation (DT), different types of exact solutions (anti-dark soliton, breather, periodic solutions, rogue wave solution).

In this paper we are going to investigate de integrability of the first version of semidiscrete cmKdV [7], focusing case, and build the multi-soliton solution. There are several ways of investigating the integrability of a dynamical system such as: a direct computation of the conserved quantities [13], the computation of Lie symmetries [14-16], the existence of the Lax pair [17, 21], building the Hirota bilinear form and computing the multi-soliton solutions $[18,19]$. We are going to use the last of the several methods listed above, tool that has gained a lot of interest in the latest few decades because of the significant results obtained [22-25] even in the supersymmetric context [26-29].

The paper is organized as follows. In Section 2, we briefly present the semidiscrete complex modified Korteweg de Vries equation and construct its Hirota bilinear form using a transformation involving two tau functions. In Section 3, we build the multi-soliton solutions using the adequate anzats. In Section 4, we perform an asymptotic analysis on the soliton solution and plot a few graphs. Last section is dedicated to conclusions.

\footnotetext{
*e-mail: babalic.corina@ucv.ro
} 


\section{The semidiscrete complex mKdV equation and the Hirota bilinear form}

The semidiscrete complex modified Korteweg-de Vries equation (semidiscrete cmKdV), introduced by Ablowitz and Ladik in [7] has the following form:

$$
\frac{d}{d t} \Phi_{n}=\left(1+\alpha\left|\Phi_{n}\right|^{2}\right)\left(\Phi_{n+1}-\Phi_{n-1}\right)
$$

where we denote by $\Phi_{n}$ the complex function $\Phi(n, t)$, where $t$ is the time variable and $n$ is the discrete space variable with step 1 . We also have to mention that $\alpha= \pm 1$ (the focusing case for $(+)$, the defocusing case for (-)).

In this paper we consider only the focusing case of the semidiscrete cmKdV equation $(\alpha=1$ in (1)):

$$
\frac{d}{d t} \Phi_{n}=\left(1+\left|\Phi_{n}\right|^{2}\right)\left(\Phi_{n+1}-\Phi_{n-1}\right)
$$

for which we apply the Hirota bilinear formalism.

Using the nonlinear substitution $\Phi_{n}=G_{n} / F_{n}$, equation becomes:

$$
\frac{D_{t} G_{n} \cdot F_{n}}{F_{n}^{2}}=\frac{F_{n}^{2}+\left|G_{n}\right|^{2}}{F_{n}^{2}} \cdot \frac{G_{n+1} F_{n-1}-G_{n-1} F_{n+1}}{F_{n+1} F_{n-1}} .
$$

Decoupling (3), we cast semidiscrete $\mathrm{cmKdV}$, focusing case, into the bilinear form:

$$
\begin{aligned}
& D_{t} G_{n} \cdot F_{n}=G_{n+1} F_{n-1}-G_{n-1} F_{n+1}, \\
& F_{n}^{2}+\left|G_{n}\right|^{2}=F_{n+1} F_{n-1},
\end{aligned}
$$

where $F_{n}$ is a real function, while $G_{n}$ is a complex valued function.

\section{The multi-soliton solution}

In order to solve this bilinear system we take for the one soliton solution the following ansatz:

$$
G_{n}=e^{\eta_{1}}, \quad F_{n}=1+e^{\eta_{1}+\eta_{1}^{*}+\phi_{13}},
$$

where $\eta_{1}=k_{1} n+\omega_{1} t+\eta_{1}^{(0)}\left(k_{1}\right.$ is the wave number, $\omega_{1}$ is the angular frequency and gives the dispersion relation and $\eta_{1}^{(0)}$ is an arbitrary phase).

From the first bilinear equation we get the dispersion relation:

$$
\omega_{1}=2 \sinh \left(k_{1}\right)
$$

while from the second we get the phase factor:

$$
e^{\phi_{13}}=\frac{1}{2\left[\cosh \left(k_{1}+k_{1}^{*}\right)-1\right]} .
$$

Explicitly, the 1-soliton solution of (1) is:

$$
\Phi_{n}=\frac{e^{k_{1} n+2 \sinh \left(k_{1}\right) t+\eta_{1}^{(0)}}}{1+\frac{1}{2\left(\cosh \left(k_{1}+k_{1}^{*}\right)-1\right)} e^{\left(k_{1}+k_{1}^{*}\right) n+2\left[\sinh \left(k_{1}\right)+\sinh \left(k_{1}^{*}\right)\right] t+\eta_{1}^{(0)}+\eta_{1}^{(0) *}} .}
$$


Straightforward calculation gives 2-soliton solution of the following form:

$$
\begin{aligned}
G_{n}= & e^{\eta_{1}}+e^{\eta_{2}}+e^{\eta_{1}+\eta_{2}+\eta_{2}^{*}+\phi_{12}+\phi_{14}+\phi_{24}}+e^{\eta_{1}+\eta_{2}+\eta_{1}^{*}+\phi_{12}+\phi_{13}+\phi_{23}} \\
F_{n}= & 1+e^{\eta_{1}+\eta_{1}^{*}+\phi_{13}}+e^{\eta_{2}+\eta_{2}^{*}+\phi_{24}}+e^{\eta_{1}+\eta_{2}^{*}+\phi_{14}}+e^{\eta_{2}+\eta_{1}^{*}+\phi_{23}}+ \\
& +e^{\eta_{1}+\eta_{1}^{*}+\eta_{2}+\eta_{2}^{*}+\sum_{1 \leq i<j}^{4} \phi_{i j}}
\end{aligned}
$$

where:

$$
\begin{gathered}
e^{\phi_{i j}}=\left\{\begin{array}{lr}
\frac{1}{2}\left[\cosh \left(k_{i}+k_{j}^{*}\right)-1\right]^{-1}, & \text { if } i=1,2 \text { and } j=3,4 ; \\
2\left[\cosh \left(k_{i}-k_{j}\right)-1\right], & \text { if } i=1,2 \text { and } j=1,2
\end{array}\right. \\
\text { or } i=3,4 \text { and } j=3,4 ;
\end{gathered}
$$

We illustrate in Figure 1 the absolute value of 1- and 2-soliton solutions of semidiscrete $\mathrm{cmKdV}$ for some chosen parameters and suitable intervals of $n, t$.

Long but straightforward calculations give the 3 -soliton solution in the form:

$$
\begin{aligned}
& G_{n}=e^{\eta_{1}}+e^{\eta_{2}}+e^{\eta_{3}}+ \\
& +e^{\eta_{1}+\eta_{2}+\eta_{2}^{*}+\phi_{12}+\phi_{15}+\phi_{25}}+e^{\eta_{2}+\eta_{1}+\eta_{1}^{*}+\phi_{12}+\phi_{14}+\phi_{24}}+e^{\eta_{3}+\eta_{1}+\eta_{1}^{*}+\phi_{13}+\phi_{14}+\phi_{34}}+ \\
& +e^{\eta_{1}+\eta_{3}+\eta_{3}^{*}+\phi_{13}+\phi_{16}+\phi_{36}}+e^{\eta_{2}+\eta_{3}+\eta_{3}^{*}+\phi_{23}+\phi_{26}+\phi_{36}}+e^{\eta_{3}+\eta_{2}+\eta_{2}^{*}+\phi_{23}+\phi_{25}+\phi_{35}}+ \\
& +e^{\eta_{1}+\eta_{2}+\eta_{3}^{*}+\phi_{12}+\phi_{16}+\phi_{26}}+e^{\eta_{1}+\eta_{3}+\eta_{2}^{*}+\phi_{13}+\phi_{15}+\phi_{35}}+e^{\eta_{2}+\eta_{3}+\eta_{1}^{*}+\phi_{23}+\phi_{24}+\phi_{34}}+ \\
& +e^{\eta_{1}+\eta_{2}+\eta_{3}+\eta_{1}^{*}+\eta_{2}^{*}+\phi_{12}+\phi_{13}+\phi_{14}+\phi_{15}+\phi_{23}+\phi_{24}+\phi_{25}+\phi_{34}+\phi_{35}+\phi_{45}}+ \\
& +e^{\eta_{1}+\eta_{2}+\eta_{3}+\eta_{1}^{*}+\eta_{3}^{*}+\phi_{12}+\phi_{13}+\phi_{14}+\phi_{16}+\phi_{23}+\phi_{24}+\phi_{26}+\phi_{34}+\phi_{36}+\phi_{46}}+ \\
& +e^{\eta_{1}+\eta_{2}+\eta_{3}+\eta_{2}^{*}+\eta_{3}^{*}+\phi_{12}+\phi_{13}+\phi_{15}+\phi_{16}+\phi_{23}+\phi_{25}+\phi_{26}+\phi_{35}+\phi_{36}+\phi_{56}} \\
& F_{n}=1+e^{\eta_{1}+\eta_{1}^{*}+\phi_{14}}+e^{\eta_{2}+\eta_{2}^{*}+\phi_{25}}+e^{\eta_{3}+\eta_{3}^{*}+\phi_{36}}+e^{\eta_{1}+\eta_{2}^{*}+\phi_{15}}+e^{\eta_{1}+\eta_{3}^{*}+\phi_{16}}+ \\
& +e^{\eta_{2}+\eta_{1}^{*}+\phi_{24}}+e^{\eta_{2}+\eta_{3}^{*}+\phi_{26}}+e^{\eta_{3}+\eta_{1}^{*}+\phi_{34}}+e^{\eta_{3}+\eta_{2}^{*}+\phi_{35}}+ \\
& +e^{\eta_{1}+\eta_{2}+\eta_{1}^{*}+\eta_{2}^{*}+\phi_{12}+\phi_{14}+\phi_{15}+\phi_{24}+\phi_{25}+\phi_{45}}+e^{\eta_{1}+\eta_{2}+\eta_{1}^{*}+\eta_{3}^{*}+\phi_{12}+\phi_{14}+\phi_{16}+\phi_{24}+\phi_{26}+\phi_{46}}+ \\
& +e^{\eta_{1}+\eta_{2}+\eta_{2}^{*}+\eta_{3}^{*}+\phi_{12}+\phi_{15}+\phi_{16}+\phi_{25}+\phi_{26}+\phi_{56}}+e^{\eta_{1}+\eta_{3}+\eta_{1}^{*}+\eta_{2}^{*}+\phi_{13}+\phi_{14}+\phi_{15}+\phi_{34}+\phi_{35}+\phi_{45}}+ \\
& +e^{\eta_{1}+\eta_{3}+\eta_{1}^{*}+\eta_{3}^{*}+\phi_{13}+\phi_{14}+\phi_{16}+\phi_{34}+\phi_{36}+\phi_{46}}+e^{\eta_{1}+\eta_{3}+\eta_{2}^{*}+\eta_{3}^{*}+\phi_{13}+\phi_{15}+\phi_{16}+\phi_{35}+\phi_{36}+\phi_{56}}+ \\
& +e^{\eta_{2}+\eta_{3}+\eta_{1}^{*}+\eta_{2}^{*}+\phi_{23}+\phi_{24}+\phi_{26}+\phi_{34}+\phi_{35}+\phi_{45}}+e^{\eta_{2}+\eta_{3}+\eta_{1}^{*}+\eta_{3}^{*}+\phi_{23}+\phi_{24}+\phi_{26}+\phi_{34}+\phi_{36}+\phi_{46}}+ \\
& +e^{\eta_{2}+\eta_{3}+\eta_{2}^{*}+\eta_{3}^{*}+\phi_{23}+\phi_{25}+\phi_{26}+\phi_{35}+\phi_{36}+\phi_{56}}+e^{\eta_{1}+\eta_{2}+\eta_{3}+\eta_{1}^{*}+\eta_{2}^{*}+\eta_{3}^{*}+\sum_{1 \leq i<j}^{6} \phi_{i j}}
\end{aligned}
$$

where:

$$
\begin{gathered}
e^{\phi_{i j}}=\left\{\begin{array}{cc}
\frac{1}{2}\left[\cosh \left(k_{i}+k_{j}^{*}\right)-1\right]^{-1}, & \text { if } i=1,2,3 \text { and } j=4,5,6 ; \\
2\left[\cosh \left(k_{i}-k_{j}\right)-1\right], & \text { if } i=1,2,3 \text { and } j=1,2,3 \\
\text { or } i=4,5,6 \text { and } j=4,5,6 ;
\end{array}\right. \\
\eta_{j}=k_{j} n+\omega_{j} t+\eta_{j}^{(0)}, \quad k_{j+3}=k_{j}^{*} \\
\omega_{j}=2 \sinh \left(k_{j}\right), \quad j=1,2,3 .
\end{gathered}
$$




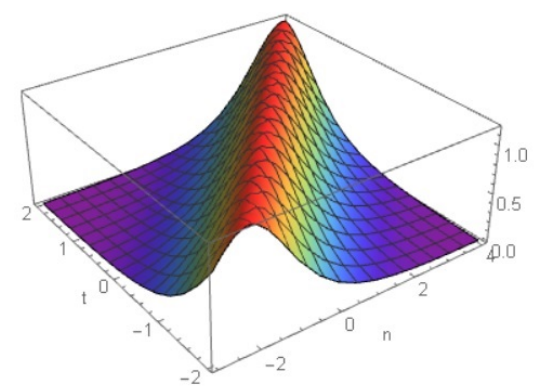

(a)

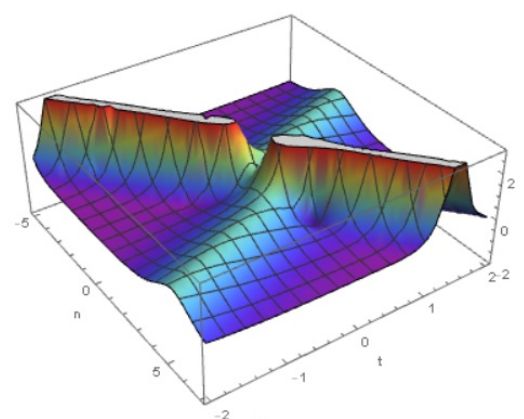

(b)

Figure 1. Plots of the absolute value $|\Phi(n, t)|^{2}$ of the soliton solutions of semidiscrete cmKdV, focusing case: 1 -ss (a) and 2-ss (b) for the chosen parameters: (a) $k_{1}=1+4 i$, (b) $k_{1}=1+i$ and $k_{2}=2+3 i$

Although the existence of three-soliton solution in Hirota form is a strong indicator for the complete integrability [20], we give here the $N$-soliton solution, which can be proved using the induction method like in [22]:

$$
\begin{aligned}
G_{n} & =\sum_{\mu=0,1} D_{2}(\underline{\mu}) \exp \left(\sum_{i=1}^{2 N} \mu_{i} \eta_{i}+\sum_{1 \leq i<j}^{2 N} \mu_{i} \mu_{j} \phi_{i j}\right) \\
F_{n} & =\sum_{\mu=0,1} D_{1}(\underline{\mu}) \exp \left(\sum_{i=1}^{2 N} \mu_{i} \eta_{i}+\sum_{1 \leq i<j}^{2 N} \mu_{i} \mu_{j} \phi_{i j}\right)
\end{aligned}
$$

where:

$$
\begin{gathered}
e^{\phi_{i j}}= \begin{cases}\frac{1}{2}\left[\cosh \left(k_{i}+k_{j}^{*}\right)-1\right]^{-1}, & \text { if } i=1, \ldots N \text { and } j=N+1, \ldots, 2 N ; \\
2\left[\cosh \left(k_{i}-k_{j}\right)-1\right], & \text { if } i=1, \ldots N \text { and } j=1, \ldots, N \\
\text { or } i=N+1, \ldots, 2 N \text { and } j=N+1, \ldots, 2 N ;\end{cases} \\
\eta_{j}=k_{j} n+\omega_{j} t+\eta_{j}^{(0)}, \quad \eta_{j+N}=\eta_{j}^{*}, \quad k_{j+N}=k_{j}^{*}, \\
\omega_{j}=2 \sinh \left(k_{j}\right), \quad \omega_{j+N}=\omega_{j}^{*}, \quad j=1, \ldots, N,
\end{gathered}
$$

and where:

$$
\begin{gathered}
D_{1}(\underline{\mu})= \begin{cases}1, & \text { when } \sum_{i=1}^{N} \mu_{i}=\sum_{i=1}^{N} \mu_{i+N} \\
0 & \text { otherwise }\end{cases} \\
D_{2}(\underline{\mu})= \begin{cases}1, & \text { when } \sum_{i=1}^{N} \mu_{i}=1+\sum_{i=1}^{N} \mu_{i+N} \\
0 & \text { otherwise }\end{cases}
\end{gathered}
$$

As a remark, the multi-soliton solutions of the focusing semidiscrete complex mKdV are very similar to those of Ablowitz-Ladik [24], only the dispersion relation is, of course, different. 


\section{Asymptotic analysis on soliton solutions}

For the semidiscrete $\mathrm{cmKdV}(1)$, the 2-ss solution has the form:

$$
\Phi_{n}=\frac{e^{\eta_{1}}+e^{\eta_{2}}+e^{\eta_{1}+\eta_{2}+\eta_{2}^{*}+\phi_{12}+\phi_{14}+\phi_{24}}+e^{\eta_{1}+\eta_{2}+\eta_{1}^{*}+\phi_{12}+\phi_{13}+\phi_{23}}}{1+e^{\eta_{1}+\eta_{1}^{*}+\phi_{13}}+e^{\eta_{2}+\eta_{2}^{*}+\phi_{24}}+e^{\eta_{1}+\eta_{2}^{*}+\phi_{14}}+e^{\eta_{2}+\eta_{1}^{*}+\phi_{23}}+e^{\eta_{1}+\eta_{1}^{*}+\eta_{2}+\eta_{2}^{*}+} \sum_{1 \leq i<j}^{4} \phi_{i j}} .
$$

In order to discuss the scattering process described, we first have to extract the initial and final states of the soliton. For this purpose we need to co-move with one of the solitons and let the other go to infinity as $|t| \rightarrow \infty$.

First we consider that we are in the co-moving frame of soliton 1 , so we have $\eta_{1}$ finit, while $\eta_{2} \rightarrow \pm \infty$.

$$
\begin{array}{ll}
\eta_{2} \rightarrow-\infty: & \Phi_{n} \rightarrow \frac{e^{\eta_{1}}}{1+e^{\eta_{1}+\eta_{1}^{*}+\phi_{13}}}, \\
\eta_{2} \rightarrow+\infty: & \Phi_{n} \rightarrow \frac{e^{\eta_{1}+\phi_{12}+\phi_{14}}}{1+e^{\eta_{1}+\eta_{1}^{*}+\phi_{12}+\phi_{13}+\phi_{14}+\phi_{23}+\phi_{34}}},
\end{array}
$$

where $e^{\phi_{i j}}$ are given in (4).

Similar, considering that we are in the co-moving frame of soliton 2 , we have $\eta_{2}$ finite, while $\eta_{1} \rightarrow \pm \infty$.

$$
\begin{array}{ll}
\eta_{1} \rightarrow-\infty: & \Phi_{n} \rightarrow \frac{e^{\eta_{2}}}{1+e^{\eta_{2}+\eta_{2}^{*}+\phi_{24}}}, \\
\eta_{1} \rightarrow+\infty: & \Phi_{n} \rightarrow \frac{e^{\eta_{2}+\phi_{12}+\phi_{23}}}{1+e^{\eta_{2}+\eta_{2}^{*}+\phi_{12}+\phi_{14}+\phi_{23}+\phi_{24}+\phi_{34}}},
\end{array}
$$

We notice that the limits differ only in the exponential, which means that the solitons are experiencing only a phase shift due to the interaction. From the asymptotic analysis of solutions, we find that the amplitudes, shapes and velocities of the 2-soliton solution remain invariant before and after the interactions, which means that their interactions are elastic and the solitons are very stable as the only changes are in their initial phases.

\section{Conclusions}

In this paper we studied the first semidiscrete version of complex mKdV equation, focusing case, the second member of the semidiscrete nonlinear Schródinger hierarchy (AblowitzLadik hierarchy). Using the Hirota bilinear formalism, we built the bilinear form and constructed the multi-soliton solutions, proving complete integrability via Hirota method. The elastic interactions between solitons and their stability is proven through asymptotic analysis.

\section{Acknowledgements:}

This work was supported by the grant POCU380/6/13/123990, co-financed by the European Social Fund within the Sectorial Operational Program Human Capital 2014 - 2020.

\section{References}

[1] R.F. Rodriguez, J.A. Reyes, A. Espinosa-Ceron, J. Fujioka and B.A. Malomed, Phys. Rev. E 68, 036606 (2003).

[2] J.S. He, L.H. Wang, L.J. Li, K. Porsezian and R. Erdélyi, Phys. Rev. E 89, 062917 (2014). 
[3] M.J. Ablowitz and P.A. Clarkson, Solitons, Nonlinear Evolution Equations and Inverse Scattering (Cambridge (University Press, New York, 1991) 516 pp.

[4] V. Makhankov, Comput. Phys. Commun. 21, 1-49 (1980).

[5] E.P. Zhidkov, I.D. Iliev and K.P. Kirchev, Sibirsk. Mat. Zh. 26, 39-47 (1985).

[6] S. Hakkaev, I.D. Iliev and K.P. Kirchev, J. Diff. Eqn. 248, 2608-2627 (2010).

[7] M. J. Ablowitz and J. F. Ladik, Journal of Mathematical Physics 17, 1011 (1976).

[8] B.M. Herbst, M.J. Ablowitz and E. Ryan, Comput. Phys. Commun.65, 137-142 (1991).

[9] T.R. Taha, Adv. Comp. Meth. for Partial Diff. Eq., VII (edited by R. Vichnevetsky, IMACS, 1992) 721-725.

[10] T.R. Taha, Math. Comp. Sim. 37 461-467 (1994).

[11] Li-Yuan Ma, Hai-Qiong Zhao, Shou-Feng Shen, Wen-Xiu Ma, Commun. Nonlin. Sci. Num. Sim. (2018).

[12] Hai-qiong Zhao and Guo-fu Yu, Chaos 27, 043113 (2017).

[13] R. Cimpoiasu and R. Constantinescu, Nonlinear Anal. Theory Methods Appl. 68(8), 2261-2268 (2008).

[14] R. Cimpoiasu and R. Constantinescu, Open Phys. 12 (2), 81-89 (2014).

[15] C. Ionescu, R. Constantinescu, M. Stoicescu, Journal of Applied Analysis and Computation 10(2), 569-583 (2020).

[16] D. J. Zhang, T. K. Ning, J. B. Bi, D. Y. Chen, Phys. Lett. A 359 (5), 458-466 (2006).

[17] C.N. Babalic, R. Constantinescu, V. Gerdjikov, Physics AUC 23, 36-41 (2013).

[18] R. Hirota, The Direct Method in Soliton (Cambridge University Press, 2004) 200 pp.

[19] R. Hirota, Chaos Solitons \& Fractals 11, 77-84 (2000).

[20] J. Hietarinta, J. Math. Phys. 28, 2094, (1987); 28, 2586 (1987).

[21] P. D. Lax, Comm. Pure Appl. Math. 21, 467-490 (1968).

[22] C.N. Babalic, A. S. Carstea, J. Phys. A: Math. Theor. 46(14), 5205 (2013).

[23] C.N. Babalic, A. S. Carstea, CEJP 12, 341-347 (2014).

[24] N.C. Babalic, A. S. Carstea, J. Phys. A: Math. Theor. 50, 41 (2017).

[25] C.N. Babalic, International Journal of Modern Physics B, 2050274 (2020).

[26] N.C. Babalic, A. S. Carstea, Theoretical and Mathematical Physics 188, 2, 1172-1180 (2016).

[27] N.C. Babalic, A. S. Carstea, J. Phys. A: Math. Theor. 51, 225204 (2018).

[28] C.N. Babalic, Physics AUC, 28, 99-110 (2018).

[29] C.N. Babalic, A.S. Carstea, Modern Physics Letters A, 2050143, (2020). 